\title{
THE INDEPENDENCE PROPERTY IN GENERALIZED DENSE PAIRS OF STRUCTURES
}

\author{
ALEXANDER BERENSTEIN, ALF DOLICH, AND ALF ONSHUUS
}

\begin{abstract}
We provide a general theorem implying that for a (strongly) dependent theory $T$ the theory of sufficiently well-behaved pairs of models of $T$ is again (strongly) dependent. We apply the theorem to the case of lovely pairs of thorn-rank one theories as well as to a setting of dense pairs of first-order topological theories.
\end{abstract}

\section{INTRODUCTION}

In this paper we study properties of theories obtained by beginning with a complete first order theory $T$ with infinite models, a model $\mathfrak{M} \models T$, and a subset $A \subset M$ and considering the pair $(\mathfrak{M}, A)$ comprised of $\mathfrak{M}$ together with a new predicate for $A$. This situation has been studied extensively, typically in the case where $A$ is an elementary submodel of $\mathfrak{M}[20]$, [3], [24] or in the context of stable theories, when the induced structure on the predicate is stable [7], [2].

Our concern here is with the independence property and pairs. Namely we consider theories $T$ without the independence property (in which case we refer to $T$ as being dependent) and then give a criterion so that a theory of pairs arising from models of $T$ also does not have the independence property. We also prove an analogous result deriving strong dependence for pairs when the original theory is strongly dependent. Our motivations for studying this problem are manifold. To begin with there has recently been considerable interest in theories without the independence property (see for example [21] or [1]) and thus is seemed appropriate to consider the properties of pairs in the context of dependent theories. On the other hand, motivation also came out of three interrelated topics: the study of lovely pairs of structures in a general context; the study of theories with o-minimal open core; and the study of first order topological theories, specifically the p-adics. We elaborate on all of these below.

The paper is structured as follows. The second section is devoted to the statement and proof of two general theorems, the first one providing a criterion under which given a dependent theory $T, \mathfrak{M} \models T$, and $A \subseteq M$ the theory of the pair $(\mathfrak{M}, A)$ is also dependent, the second one a criterion for the pair $(\mathfrak{M}, A)$ to be strongly dependent assuming that $T$ is strongly dependent. The next two sections are devoted to examples where the theorem applies; the first example is lovely pairs of theories where acl has the exchange property, studied in section 3 , and the second is a class of first order topological theories studied in section 4 .

Date: September 2008.

1991 Mathematics Subject Classification. 03C45.

Key words and phrases. rosy theories, lovely pairs, dense pairs, dependent theories, strongly dependent theories. 
Lovely pairs have been studied from many points of view. They were first defined by Poizat [20] in the stable setting and used by Buechler to study pregeometries associated to strongly minimal sets [6]. The idea was generalized by Vassiliev [24] to the setting of rank one simple theories in order to study their pregeometries. Ben Yaacov, Pillay and Vassiliev [3] worked out the theory of pairs in the general framework of simple theories. Vassiliev's original setting can be easily modified to deal with theories where the algebraic closure satisfies the exchange property (see [4]), this new framework includes the rank one simple theories, o-minimal theories and b-rank one rosy theories. As before, the pair provides information about the underlying pregeometries in the o-minimal case [4]. The lovely pairs of o-minimal structures had been studied already by van den Dries as dense pairs of o-minimal structures [10].

Many properties of the original theory are preserved in the associated theory of lovely pairs, such as (super) stability and (super) simplicity. The aim of section 3 is to show that if the original theory is (strongly) dependent, then the corresponding theory of lovely pairs is again (strongly) dependent. Similar results have been obtained independently by Boxall (for lovely pairs of real closed fields) and by Günaydin and Hieronymi [15]. For this section we assume the reader is familiar with basic ideas of rosy theories and dependent theories, we refer the reader to $[1,19]$ and [23].

A specific example of a lovely pair of thorn-rank one structures is a dense pair of o-minimal structures as studied by van den Dries in [10]. Specifically if $T$ is an o-minimal theory extending the theory of an ordered abelian group, $\mathfrak{N}=T$, and $\mathfrak{M} \prec \mathfrak{N}$ with $M$ dense in $N$, then the pair $(\mathfrak{N}, \mathfrak{M})$ is a model of the corresponding theory of lovely pairs (see [4]). Thus our main theorem applies and the theory of this pair is dependent. This result was of primary interest to the second author in light of work on o-minimal open cores (see [9]). The theory of a dense pair of o-minimal structures provides a canonical example of a theory with all of whose models have o-minimal open core as do "generic" expansions of o-minimal theories (see [8]). Arguably the dense pairs are the more "natural" of these examples. In [9] a host of desirable model theoretic properties are studied in the context of ominimal open cores and for the vast majority of these the generic expansions have the property while the dense pairs do not. Thus our main theorem establishes that at least in so far as the independence property is concerned dense pairs have the desirable property (dependence) while it is well known that generic expansions have the independence property under very weak assumptions.

Finally given dense pairs of o-minimal structures as a starting point it is natural to ask whether similar results hold in other structures with a definable topology, for example the theory of the p-adic field. We use this opportunity to develop in a general context of first order topological structures (inspired by the work in [18]) much of the machinery of dense pairs found in [10]. Given these results we then may apply our main theorem to show that if the theory we begin with is dependent then so is the resultant theory of dense pairs. Specifically we prove that we have a good theory for dense pairs of p-adic fields and the theory of the such dense pairs is dependent. 


\section{The Context and The Main Theorem}

Throughout this section we consider $\mathcal{L}$-structures $\mathfrak{M}$ with theory $T$ that for convenience we assume has quantifier elimination. We consider an expansion of $\mathcal{L}$ by a new unary predicate $P$ which we denote by $\mathcal{L}_{P}$. For $A \subseteq M$ we write $(\mathfrak{M}, A)$ for the $\mathcal{L}_{P}$-structure obtained from $\mathfrak{M}$ by interpreting $P$ as $A$. We will denote its theory by $T_{A}$.

Notation 2.1. With the above notation for $B \subseteq M$ we write $P(B)$ for $B \cap A$. In particular note that $P(M)=A$.

Notation 2.2. Let $(\mathfrak{M}, A)$ be a pair as above. Throughout this paper, acl, $\operatorname{acl}_{P}$ stand for the algebraic closure in the languages $\mathcal{L}, \mathcal{L}_{P}$ respectively. We write $\operatorname{tp}(\vec{b})$, $\mathrm{qftp}(\vec{b}), \operatorname{tp}_{P}(\vec{b}), \operatorname{qftp}_{P}(\vec{b})$ for types and quantifier free types in the languages $\mathcal{L}, \mathcal{L}_{P}$ respectively. When $(M$, acl $)$ is a pregeometry independence means acl-independent, dimension is considered in terms of $(M$, acl).

Assumption 2.3. Throughout this section we assume that for any $T$ we consider acl satisfies exchange and that any subset $A \subseteq M$ we choose to name for the predicate is acl-closed.

Definition 2.4. Given $T, \mathfrak{M} \models T$, and $A \subseteq M$ we may form the $\mathcal{L}_{P^{-}}$-structure $(\mathfrak{M}, A)$. We say that $B \subseteq M$ is $P$-independent if $B$ is algebraically independent from $P(M)$ over $P(B)$.

The next definition is key, we isolate the essential property of a subset of a model so that upon naming it by a predicate the resulting theory is still well-behaved.

Definition 2.5. We say that $A$ is innocuous if whenever $\left(\mathfrak{M}_{0}, A_{0}\right),\left(\mathfrak{M}_{1}, A_{1}\right) \models T_{A}$, $\vec{a}_{0} \in M_{0}, \vec{a}_{1} \in M_{1}$ are both $P$-independent, and $\operatorname{qftp}_{P}\left(\vec{a}_{0}\right)=\operatorname{qftp}_{P}\left(\vec{a}_{1}\right)$ then $t p_{P}\left(\vec{a}_{0}\right)=t p_{P}\left(\vec{a}_{1}\right)$.

The following is a key property of innocuous sets, see for example Proposition 3.2 in [4] for a proof of how this follows from $A$ being innocuous.

Observation 2.6. If $A$ is innocuous and $(\mathfrak{N}, B) \models T_{A}$ then any $\mathcal{L}_{P}$-definable $X \subseteq B$ is simply the intersection of an $\mathcal{L}$-definable subset of $N$ with $P(N)$.

Now we prove our main result:

Theorem 2.7. Let $T$ be a dependent theory in a language $\mathcal{L}$ so that acl satisfies the exchange property on models of $T$. Let $\mathfrak{M} \models T$ and suppose that $A \subseteq M$ is innocuous and $\operatorname{acl}(A)=A$. Then the theory of the pair $(\mathfrak{M}, A)$ is also dependent.

Proof. Assume $T_{A}$ has the independence property. Let $(\mathfrak{M}, A) \models T_{A}$ be a monster model, let $\kappa=2^{|\mathcal{L}|}$, let $\left\{a_{i}: i \in \kappa\right\}$ be an $\mathcal{L}_{P}$-indiscernible sequence and assume there is an $\mathcal{L}_{P}$-formula $\varphi(x, \vec{y})$ such that for any $I, J \subset \kappa$ disjoint, there is $\vec{b}_{I J} \in M$ such that $\varphi\left(a_{i}, \vec{b}_{I J}\right)$ holds for $i \in I$ and $\neg \varphi\left(a_{i}, \vec{b}_{I J}\right)$ holds for $i \in J$.

Note that $\left\{a_{i}: i \in \kappa\right\}$ is an $\mathcal{L}$-indiscernible sequence.

Claim $\left\{a_{i}: i \in \kappa\right\}$ is an acl-independent sequence.

Otherwise, as acl satisfies the exchange property, we have for some $n$ that $a_{n} \in$ $\operatorname{acl}\left\{a_{i}: i<n\right\}$ and there is an algebraic formula $\chi\left(x, a_{0}, \ldots, a_{n-1}\right)$ true for $a_{n}$. Since the sequence is indiscernible then this formula holds for all $a_{i}$ with $i \geq n$ and thus $a_{i}=a_{n}$ for $i>n$. This implies that $\left\{a_{i}: i \in \kappa\right\}$ is a constant sequence and it can not be a witness for the independence property, a contradiction. 
Let $\vec{b}$ be such that $\varphi\left(a_{i}, \vec{b}\right)$ if and only if $i$ is odd.

Claim $a_{i} \in M \backslash P(M)$

Otherwise $a_{i} \in P(M)$ for each $i \in \kappa$. Since $A$ is innocuous the definable subsets of $P(M)$ are intersections of the $\mathcal{L}$-definable subsets of $M$ with $P(M)$. So there is an $\mathcal{L}$-formula $\psi(x, \vec{z})$ such that $\varphi(x, \vec{b}) \wedge P(x)=\psi(x, \vec{c}) \wedge P(x)$ for some $\vec{c}$. Thus $\psi\left(a_{i}, \vec{c}\right)$ holds if and only if $i$ is odd and the $\mathcal{L}$-formula $\psi(x, \vec{z})$ has the independence property, a contradiction.

We consider two cases:

Case 1: There is no $\vec{d}$ such that $a_{i} \in \operatorname{acl}(P(M), \vec{d})$ for cofinally many $i$. In a naïve sense, we are dealing the case when $\left\{a_{i}: i \in \kappa\right\}$ is a $\mathcal{L}_{P}$-Morley sequence of generics. Making $\vec{b}$ larger if necessary, we may assume that $\vec{b}$ is $P$-independent. By our assumption, we can find a set of indices $I_{0}$ such that $I_{0}$ is not cofinal in $\kappa$ and $a_{i} \notin \operatorname{acl}(\vec{b}, P(M))$ for $i \in \kappa \backslash I_{0}$. Note that the tuple $\vec{b} a_{i}$ is also $P$-independent for all $i \notin I_{0}$. Let $\Phi(x, \vec{y})$ be a partial $\mathcal{L}_{P}$-type such that $\models \Phi\left(a_{i}, \vec{b}\right)$ for $i \notin I_{0}$ and whenever $\models \Phi\left(c, \vec{b}^{\prime}\right)$ holds, we have that $\operatorname{tp}_{P}(\vec{b})=\operatorname{tp}_{P}\left(\vec{b}^{\prime}\right)$ (in particular $\vec{b}^{\prime}$ is $P$-independent) and $c \notin \operatorname{acl}\left(P(M), \vec{b}^{\prime}\right)$. Since $A$ is innocuous for any $c, d \models \Phi(x, \vec{b})$, $\operatorname{tp}_{P}(c, \vec{b})=\operatorname{tp}_{P}(d, \vec{b})$ if and only if $\operatorname{qftp}_{P}(c, \vec{b})=\operatorname{qftp}_{P}(d, \vec{b})$. Thus by compactness, we can find a quantifier free $\mathcal{L}_{P}$-formula $\psi(x, \vec{y})$ such that for any $c \models \Phi(x, \vec{b})$, $\models \varphi(c, \vec{b})$ if and only if $=\psi(c, \vec{b})$. Let $i \in \kappa \backslash I_{0}$, then $\models \psi\left(a_{i}, \vec{b}\right)$ holds iff $i$ is odd.

So it remains to show that quantifier free $\mathcal{L}_{P}$-formulas on do not have the independence property. The result is clear for quantifier free $\mathcal{L}$-formulas and since formulas with the Independence Property are closed under boolean combinations, the problem reduces to show that formulas of the form $\psi(x, \vec{b})=P(t(x, \vec{b}))$, where $t(x, \vec{y})$ is a term, do not have the Independence Property. So assume that for $i \in \kappa \backslash I_{0}$, $P\left(t\left(a_{i}, \vec{b}\right)\right)$ holds if and only if $i$ is odd. Since the tuple $a_{i} \vec{b}$ is $P$-independent, for $i$ odd $t\left(a_{i}, \vec{b}\right) \in \operatorname{acl}(P(\vec{b}))$. We may write $\vec{b}=\left(b_{1}, \ldots, b_{l}, b_{l+1}, \ldots, b_{n}\right)$, where $P(\vec{b})=$ $\left(b_{1}, \ldots, b_{l}\right)$. There is a single algebraic $\mathcal{L}$-formula $\rho\left(x, b_{1}, \ldots, b_{l}\right)$ and $I_{1} \subset \kappa \backslash I_{0}$ infinite such that $\rho\left(t\left(a_{i}, \vec{b}\right), b_{1}, \ldots b_{l}\right)$ holds for $i \in I_{1}$. Note that since $\rho\left(x, b_{1}, \ldots, b_{l}\right)$ is algebraic and $A$ is algebraically closed, all the realizations belong to $P$ and thus $\left.\neg \rho\left(t\left(a_{i}, \vec{b}\right), b_{1}, \ldots b_{l}\right)\right)$ holds for even $i$. Thus the $\mathcal{L}$-formula $\left.\rho\left(t(x, \vec{b}), b_{1}, \ldots b_{l}\right)\right)$ has the independence property, a contradiction.

Case 2: There is $\vec{d}$ such that $a_{i} \in \operatorname{acl}(P(M), \vec{d})$ for cofinally many $i$. Since $\kappa=2^{|\mathcal{L}|}$ there is a single formula $\theta(x, \vec{y}, \vec{z}), I_{0} \subset \kappa$ cofinal and tuples $\left\{\vec{c}_{i}: i \in I_{0}\right\} \subset$ $P(M)$ such that $\theta\left(x, \vec{c}_{i}, \vec{d}\right)$ is algebraic for all $i \in I_{0}$ and $\theta\left(a_{i}, \vec{c}_{i}, \vec{b}\right)$ holds for all $i \in I_{0}$. Thus, exchanging the original sequence $\left\{a_{i}: i \in \kappa\right\}$ for $\left\{a_{i}: i \in I_{0}\right\}$ we may assume that the elements in the sequence $\left\{a_{i}: i \in \kappa\right\}$ are algebraic over $\overrightarrow{d P}(M)$ and that the algebraic formula $\theta\left(x, \vec{c}_{i}, \vec{d}\right)$ isolates $\operatorname{tp}\left(a_{i} / \vec{c}_{i}, \vec{d}\right)$. Furthermore, we may assume there is $n \geq 1$ such that $\exists \leq n x \theta\left(x, \vec{c}_{i}, \vec{d}\right)$ for all $i$.

Now let $\lambda$ be larger than $\beth_{\left(2^{|T|} \mid+\right.}$ and enlarge $\left\{\left(a_{i} \vec{c}_{i} \vec{d}\right): i \in \kappa\right\}$ to a sequence of length $\lambda$. By an Erdös-Rado argument(the method used to prove Morley's omitting types theorem), there is an indiscernible sequence $\left\{\left(a_{i}^{\prime} \vec{c}_{i}^{\prime} \vec{d}^{\prime}\right): i \in \omega\right\}$ such that for any $n$ there are $i_{1}<i_{2}<\cdots<i_{n} \in \lambda$ such that

$$
\operatorname{tp}\left(a_{i_{1}}, \vec{c}_{i_{1}}, \vec{d}, \ldots, a_{i_{n}}, \vec{c}_{i_{n}}, \vec{d}\right)=\operatorname{tp}\left(a_{1}^{\prime} \vec{c}_{1}^{\prime} \vec{d}^{\prime}, \ldots, a_{n}^{\prime} \vec{c}_{n}^{\prime} \vec{d}^{\prime}\right) .
$$

In particular, $\operatorname{tp}\left(a_{i}: i<\omega\right)=\operatorname{tp}\left(a_{i}^{\prime}: i<\omega\right)$. Again we may exchange $\left\{a_{i}: i \in \omega\right\}$ for $\left\{a_{i}^{\prime}: i \in \omega\right\}$ and assume that $\left\{\left(a_{i} \overrightarrow{c_{i}} \vec{d}\right): i \in \omega\right\}$ is an indiscernible sequence. 
We work now with the sequence $\left\{a_{i} c_{i} \vec{d}: i \in \omega\right\}$ and we assume there is $\vec{b}$ such that $\varphi\left(a_{i}, \vec{b}\right)$ holds if and only if $i$ is odd. Making the tuple $\vec{b}$ larger if necessary, we may assume that $\overrightarrow{d b}$ is $P$-independent. Note that for all $i$, the tuple $\overrightarrow{d b} \vec{c}_{i} a_{i}$ is also $P$-independent. Let $\Phi(x, \vec{y}, \vec{z}, \vec{w})$ be a partial $\mathcal{L}_{P}$-type such that $=\Phi\left(a_{i}, \vec{c}_{i}, \vec{d}, \vec{b}\right)$ for $i \in \omega$ and whenever $=\Phi\left(a^{\prime}, \vec{c}^{\prime}, \vec{d}^{\prime}, \vec{b}^{\prime}\right)$ holds, then we have that $\operatorname{tp}_{P}(\overrightarrow{d \vec{b}})=\operatorname{tp}_{P}\left(\vec{d}^{\prime} \vec{b}^{\prime}\right), \vec{c}^{\prime} \in P(M), \theta\left(a^{\prime}, \vec{c}^{\prime}, \vec{d}^{\prime}\right)$ holds and $\exists \leq n x \theta\left(x, \vec{c}^{\prime}, \vec{d}^{\prime}\right)$. In particular, all realizations of $\Phi(x, \vec{y}, \vec{z}, \vec{w})$ are $P$-independent. Since $A$ is innocuous, for any $(e, \vec{f}),\left(e^{\prime}, \vec{f}^{\prime}\right)=\Phi(x, \vec{y}, \vec{d}, \vec{b}), \operatorname{tp}_{P}(e, \vec{f}, \vec{d}, \vec{b})=\operatorname{tp}_{P}\left(e^{\prime}, \overrightarrow{f^{\prime}}, \vec{d}, \vec{b}\right)$ if and only if $\operatorname{qftp}_{P}(e, \vec{f}, \vec{d}, \vec{b})=\operatorname{qftp}_{P}\left(e^{\prime}, \vec{f}^{\prime}, \vec{d}, \vec{b}\right)$. By compactness, we can find a quantifier free $\mathcal{L}_{P}$-formula $\psi(x, \vec{y}, \vec{z}, \vec{w})$ such that for any $a^{\prime} \vec{c}^{\prime}=\Phi(x, \vec{w}, \vec{b}, \vec{d})$, $=\varphi\left(a^{\prime}, \vec{b}\right)$ if and only if $\models \psi\left(a^{\prime}, \vec{c}^{\prime}, \vec{b}, \vec{d}\right)$. So we get a quantifier free $\mathcal{L}_{P}$-formula witnessing the independence property. As before, the problem reduces to showing that formulas of the form $\psi(x, \vec{z}, \vec{b}, \vec{d})=P(t(x, \vec{z}, \vec{b}, \vec{d}))$, where $t(x, \vec{z}, \vec{y}, \vec{w})$ is a term, do not have the Independence Property. We may assume the sequence has length $|T|^{+}$instead of $\omega$. Since each tuple $a_{i} \overrightarrow{c_{i}} \vec{b} \vec{d}$ is $P$-independent, for odd $i$ we have that $t\left(a_{i}, \vec{c}_{i}, \vec{b}, \vec{d}\right) \in \operatorname{acl}\left(\vec{c}_{i}, b_{1}, \ldots, b_{l}, d_{1}, \ldots, d_{t}\right)$, where $\left(d_{1}, \ldots d_{t}\right)=P(\vec{d})$, $\left(b_{1}, \ldots, b_{l}\right)=P(\vec{b})$. Since $|T|^{+}>|T|$, there is $I_{1} \subset|T|^{+}$infinite and a single formula $\rho\left(x, \vec{z}, b_{1}, \ldots, b_{l}, d_{1}, \ldots, d_{t}\right)$ which is algebraic for any choice of $\vec{z}$ such that $\rho\left(t\left(a_{i}, \vec{c}_{i}, \vec{b}, \vec{d}\right), \vec{c}_{i}, b_{1}, \ldots b_{l}, d_{1}, \ldots, d_{t}\right)$ holds for $i \in I_{1}$. Since the formula

$$
\rho\left(x, \vec{z}, b_{1}, \ldots, b_{l}, d_{1}, \ldots, d_{t}\right)
$$

is algebraic for any choice of $\vec{z}$, we have for any $i$ that the sets of realizations of $\left.\rho\left(x, \vec{c}_{i}, b_{1}, \ldots, b_{l}, d_{1}, \ldots, d_{t}\right)\right)$ belong to $P$ and thus

$$
\neg \rho\left(t\left(a_{i}, \vec{c}_{i}, \vec{b}, \vec{d}\right), b_{1}, \ldots, b_{l}, d_{1}, \ldots, d_{t}\right)
$$

holds for even $i$. Thus the $\mathcal{L}$-formula $\left.\rho\left(t(x, \vec{z}, \vec{b}, \vec{d}), b_{1}, \ldots b_{l}, d_{1}, \ldots, d_{t}\right)\right)$ has the independence property, a contradiction.

We now consider the special case where $T$ is strongly dependent. We recall the definition (see [22] for details).

Definition 2.8. A theory $T$ is strongly dependent if we may not find $\mathfrak{M} \models T$, formulas $\phi_{i}(\vec{x}, \vec{y})$ for $i \in \omega$, elements $\vec{a}_{i}^{j} \in M$ so that for each $\eta: \omega \rightarrow \omega$ the type $p_{\eta}(\vec{x})$ :

is consistent.

$$
\bigwedge_{\{i, j \in \omega: \eta(i)=j\}} \phi_{i}\left(\vec{x}, \vec{a}_{i}^{j}\right) \wedge \bigwedge_{\{i, j \in \omega: \eta(i) \neq j\}} \neg \phi\left(\vec{x}, \vec{a}_{i}^{j}\right)
$$

Recall the following fact from [22].

Fact 2.9. The following conditions are equivalent for a complete theory $T$.

(1) $T$ is strongly dependent.

(2) We may not find $\mathfrak{M}=T$, formulas $\varphi_{i}(\vec{x}, \vec{y})$ for $i \in \omega$ and a sequence $\left\{\vec{a}_{k}^{i}\right.$ : $k<\omega, i<\omega\}$ of elements of $M$ so that $\left\{\vec{a}_{k}^{i}: k<\omega\right\}$ is indiscernible over $\left\{\vec{a}_{k}^{j}: j<k, j \neq i, k<\omega\right\}$ for each $i<\omega$ and $\left\{\varphi\left(\vec{x}, \vec{a}_{0}^{i}\right) \wedge \neg \varphi\left(\vec{x}, \vec{a}_{1}^{i}\right): i<\omega\right\}$ is consistent.

(3) For every indiscernible sequence $\left(\vec{b}_{i}\right)_{i<\kappa}$ with tuples $\vec{b}_{i}$ that are at most countable, and every finite set $C$, there is a convex equivalence relation $\sim$ 
on $\kappa$ with finitely many classes and such that $\operatorname{tp}\left(\vec{b}_{i} / C\right)$ only depends on the $\sim$-class of $i$.

(4) For every indiscernible sequence $\left(\vec{b}_{i}\right)_{i \in I}$ with tuples $\vec{b}_{i}$ that are at most countable, and every finite set $C$, there is a convex equivalence relation $\sim$ on I with finitely many classes and such that $\left(\vec{b}_{i}\right)_{i \in j / \sim}$ is indiscernible over $C$.

Furthermore, in clauses (3), (4) it is enough to consider the case where $C$ is a singleton.

Theorem 2.10. Let $T$ be a strongly dependent theory in a language $\mathcal{L}$ so that acl satisfies the exchange property on models of $T$. Let $\mathfrak{M} \models T$ and suppose that $A \subseteq M$ is innocuous and $\operatorname{acl}(A)=A$. Then the theory of the pair $(\mathfrak{M}, A)$ is also strongly dependent.

Proof. Let $\left(\vec{b}_{i}\right)_{i<\kappa}$ be an $\mathcal{L}_{P}$-indiscernible sequence, where the tuples $\vec{b}_{i}$ that are at most countable and let $a \in M$ be an element. We will use Fact 2.9(3), so we want to show that there is a convex equivalence relation $\sim$ on $\kappa$ with finitely many classes such that $\operatorname{tp}_{P}(\vec{b} / a)$ only depends on $\sim$-class of $i$. We may assume after enlarging each $\vec{b}_{i}$ if necessary and using an Erdös-Rado argument, that each $\vec{b}_{i}$ is $P$-independent. We divide our work by cases:

Case 1. $a \in P(M)$

We prove the result for a tuple $\vec{a} \in P(M)^{k}$. Note that the tuples $\vec{b}_{i} \vec{a}$ are again $P$-independent. Since the sequence $\left(\vec{b}_{i}\right)_{i<\kappa}$ is an $\mathcal{L}$-indiscernible sequence, there is an equivalence relation $\sim$ on $\kappa$ with finitely many convex classes and such that $\operatorname{tp}\left(\vec{b}_{i} / \vec{a}\right)$ only depends on the $\sim$-class of $i$. Our goal is to prove that $\operatorname{tp}_{P}\left(\vec{b}_{i} / \vec{a}\right)$ only depends on the $\sim$-class of $i$. Since $\vec{b}_{i} \vec{a}$ is $P$-independent, it suffices to show that $\mathrm{qftp}_{p}\left(\vec{b}_{i} / \vec{a}\right)$ is the same for all $\sim$-classes and in order to prove this, it is enough to check that for a term $t\left(\vec{b}_{i}, a\right)$, the value of $P\left(t\left(\vec{b}_{i}, \vec{a}\right)\right)$ is constant on each class. Assume that $P\left(t\left(\vec{b}_{i}, \vec{a}\right)\right)$ holds for some $i$ and that $i \sim j$. Since $\vec{b}_{i} \vec{a}$ is $P$ independent, $t\left(\vec{b}_{i}, \vec{a}\right) \in \operatorname{acl}(P(\vec{b} \vec{a}))=\operatorname{acl}(P(\vec{b}) \vec{a})$ and there is an algebraic $\mathcal{L}$-formula $\theta\left(x, \vec{a}, P\left(\vec{b}_{i}\right)\right)$ such that $\theta\left(t\left(\vec{b}_{i}, \vec{a}\right), \vec{a}, P\left(\vec{b}_{i}\right)\right)$ holds. Since $\operatorname{tp}\left(\vec{b}_{i} / \vec{a}\right)=\operatorname{tp}\left(\vec{b}_{j} / \vec{a}\right)$ we have that $\theta\left(t\left(\vec{b}_{j}, \vec{a}\right), \vec{a}, P\left(\vec{b}_{j}\right)\right)$ also holds and since $P$ is algebraically closed, we get that $P\left(t\left(\vec{b}_{j}, \vec{a}\right)\right)$ holds as well.

Case 2. $a \in \operatorname{acl}\left(P(M),\left(\vec{b}_{i}\right)_{i<\kappa}\right)$

Then there are $t_{1}, \ldots, t_{m} \in P(M)$ and $\vec{b}_{i_{1}}, \ldots, \vec{b}_{i_{l}} \in\left(\vec{b}_{i}\right)_{i<\kappa}$ such that $a \in$ $\operatorname{acl}\left(\vec{b}_{i_{1}}, \ldots, \vec{b}_{i_{l}}, t_{1}, \ldots, t_{m}\right)$. First consider $\sim_{1}$ defined on $\kappa$ by $k \sim_{1} j$ if $k, j$ are in the same interval defined by $i_{1}, \ldots, i_{l}$. For each $i$, we have $\left(\vec{b}_{j} \vec{b}_{i_{1}}, \ldots, \vec{b}_{i_{l}}\right)_{j \in i / \sim}$ is an indiscernible sequence and the $\sim_{1}$-classes are convex. Fix $i \in \kappa$ such that $i / \sim$ is infinite and we work now with the sequence $\left(\vec{b}_{j} \vec{b}_{i_{1}}, \ldots, \vec{b}_{i_{l}}\right)_{j \in i / \sim}$. Enlarging the $\vec{b}_{j}$ 's if necessary and using an Erdös-Rado argument, we may assume that $\left(\vec{b}_{j} \vec{b}_{i_{1}}, \ldots, \vec{b}_{i_{l}}\right)$ is $P$-independent for $j \in i / \sim$. Since $T$ is strongly dependent there is an equivalence relation $\sim$ that refines $\sim_{1}$ also with finitely many equivalence classes, each of which is convex, such that for each $j$ we have $\operatorname{tp}\left(\vec{b}_{k}, \vec{b}_{i_{1}}, \ldots, \vec{b}_{i_{l}}, t_{1}, \ldots, t_{m}, a\right)=$ $\operatorname{tp}\left(\vec{b}_{j}, \vec{b}_{i_{1}}, \ldots, \vec{b}_{i_{l}}, t_{1}, \ldots, t_{m}, a\right)$ whenever $k \sim j$. As in the previous case we have, by transitivity, that each tuple $\left(\vec{b}_{k}, \vec{b}_{i_{1}}, \ldots, \vec{b}_{i_{l}}, t_{1}, \ldots, t_{m}\right)$ is $P$-independent, so $\left(\vec{b}_{k}, \vec{b}_{i_{1}}, \ldots, \vec{b}_{i_{l}}, t_{1}, \ldots, t_{m}, a\right)$ is also $P$-independent and thus it suffices to prove 
that $\operatorname{qftp}_{P}\left(\vec{b}_{k}, \vec{b}_{i_{1}}, \ldots, \vec{b}_{i_{l}}, t_{1}, \ldots, t_{m}, a\right)=\operatorname{qftp}_{P}\left(\vec{b}_{j}, \vec{b}_{i_{1}}, \ldots, \vec{b}_{i_{l}}, t_{1}, \ldots, t_{m}, a\right)$. This is checked as in case 1 .

Case 3. $a \notin \operatorname{acl}\left(P(M),\left(\vec{b}_{i}\right)_{i<\kappa}\right)$

As in case 1 , the tuples $\vec{b}_{i} a$ are again $P$-independent. Since the sequence $\left(\vec{b}_{i}\right)_{i<\kappa}$ is an $\mathcal{L}$-indiscernible sequence, there is an equivalence relation $\sim$ on $\kappa$ with finitely many convex classes and such that $\operatorname{tp}\left(\vec{b}_{i} / \vec{a}\right)$ only depends on the $\sim$-class of $i$. Our goal is to prove that $\operatorname{tp}_{p}\left(\vec{b}_{i} / \vec{a}\right)$ only depends on the $\sim$-class of $i$. Since $\vec{b}_{i} a$ is $P$ independent, it suffices to check that for a term $t\left(\vec{b}_{i}, a\right)$, the value of $P\left(t\left(\vec{b}_{i}, a\right)\right)$ is constant on each $\sim$ class. Assume that $P\left(t\left(\vec{b}_{i}, a\right)\right)$ holds for some $i$ and that $i \sim j$. Since $\vec{b}_{i} a$ is $P$-independent, there is an algebraic $\mathcal{L}$-formula $\theta\left(x, P\left(\vec{b}_{i}\right)\right)$ such that $\theta\left(t\left(\vec{b}_{i}, a\right), P\left(\vec{b}_{i}\right)\right)$ holds. Since $\operatorname{tp}\left(\vec{b}_{i} / a\right)=t p\left(\vec{b}_{j} / a\right)$ we have that $\theta\left(t\left(\vec{b}_{j}, a\right), P\left(\vec{b}_{j}\right)\right)$ also holds and since $P$ is algebraically closed, we get that $P\left(t\left(\vec{b}_{j}, a\right)\right)$ also holds.

\section{Lovely Pairs of Theories with the Exchange Property}

In this section we give our first application of Theorem 2.7 to the case of lovely pairs associated to theories where the algebraic closure satisfies the exchange property. Let $T$ be a complete theory in a language $\mathcal{L}$ with quantifier elimination that eliminates the quantifier $\exists^{\infty}$ and such that acl satisfies exchange. Examples of such theories includes $S U$-rank one simple theories with quantifier elimination, O-minimal theories extending DLO and thorn-rank one theories with quantifier elimination and eliminating $\exists^{\infty}$ (see [19] for details on thorn-rank and related issues).

For the ensuing we let $T^{\prime}$ be the theory of pairs $(\mathfrak{M}, P(M))$ where $\mathfrak{M}=T$ and $P(M)$ is algebraically closed in $M$.

Definition 3.1. We say that a structure $(\mathfrak{M}, P(M))$ is a lovely pair of models of $T$ if

(1) $(\mathfrak{M}, P(M)) \models T^{\prime}$

(2) (Coheir or density property) If $A \subset M$ is algebraically closed and finite dimensional and $q \in S_{1}(A)$ is non-algebraic $\mathcal{L}$-type, there is $a \in P(M)$ such that $a \models q$.

(3) (Extension property) If $A \subset M$ is algebraically closed and finite dimensional and $q \in S_{1}(A)$ is non-algebraic $\mathcal{L}$-type, there is $a \in M, a \models q$ and $a \notin$ $\operatorname{acl}(A \cup P(M))$.

It is proved in [4] that two lovely pairs of $T$ are elementarily equivalent. We write $T_{P}$ for their common complete theory. It is also proved in [4] that since $T$ eliminates the quantifier $\exists^{\infty}$ the saturated models of $T_{P}$ are again lovely pairs. The proofs are a straightforward generalization of the corresponding results from [24] where the underlying theory $T$ is simple of rank one.

Recall that a subset $B \subseteq M$ is $P$-independent if $B$ is algebraically independent from $P(M)$ over $P(B)$. The main property of $P$-independent sets is (see [4, Lemma 2.6], [3, Lemma 3.8]):

Theorem 3.2. Let $T$ be a complete theory where acl satisfies exchange and that eliminates $\exists^{\infty}$. If $(\mathfrak{M}, P(M))$ is a lovely pair of models of $T$ then $P(M)$ is an innocuous subset of $M$.

Corollary 3.3. If $T$ is a complete dependent theory where acl satisfies exchange, that has quantifier elimination and that eliminates $\exists^{\infty}$, then the theory of lovely 
pairs of models of $T$ is also dependent. Moreover, if $T$ is strongly dependent, then the corresponding theory of lovely pairs $T_{P}$ is strongly dependent.

Proof. We apply Theorem 2.7. If $(\mathfrak{M}, P(M))$ is lovely then the previous theorem yields that $P(M)$ is innocuous in $M$. For the "moreover" part apply Theorem 2.10 .

In particular, the theory of lovely pairs of thorn-rank one dependent theories that eliminate the quantifier $\exists^{\infty}$ is again dependent.

\section{Dense Paris and the P-Adics}

In this section we consider properties of pairs of structures $(\mathfrak{N}, \mathfrak{M})$ with $\mathfrak{M} \prec \mathfrak{N}$ (notice we insist that this is a proper inclusion) where, most typically, $\mathfrak{M}$ and $\mathfrak{N}$ are expansions of fields. Our primary interest is in the case where $\mathfrak{M}$ is a model of the theory of the p-adics $\mathbb{Q}_{p}$ in the language of fields. Throughout $T$ will denote a complete theory expanding that of integral domains in a language $\mathcal{L}$. $P$ will be a new unary predicate and $\mathcal{L}_{P}$ denotes $\mathcal{L}$ augmented by $P$. We begin with some observations originally motivating this part of our study.

It is a simple yet useful observation (originally due to van den Dries) that the real closed field $\overline{\mathbb{R}}=\langle\mathbb{R},+, \cdot,<\rangle$ is the unique real closed field so that every elementary submodel of $\overline{\mathbb{R}}$ is dense in $\overline{\mathbb{R}}$ and every elementary extension of $\overline{\mathbb{R}}$ is tame (recall that $R_{1} \preceq R_{2}$ is a tame extension of real closed fields if for every $x \in R_{2}$ in the convex hull of $R_{1}$ there is a unique $y \in R_{1}$ so that $|x-y|<z$ for every $z \in R_{1}^{>0}$ ). This observation makes it natural to study two genres of pairs of real closed fields (or more generally o-minimal theories): dense pairs (as in [10]) or tame pairs (as in [12]).

Considering the usual analogy between real closed fields and p-adically closed fields we may try to treat the p-adic fields $\mathbb{Q}_{p}$ similarly. Of course we need to first develop a reasonable analog for tameness in this context. For the ensuing definition given a valued field $K$ we write $\Gamma(K)$ for its value group and $v$ for the valuation.

Definition 4.1. Given $p$-adically closed fields $K \preceq L$ we say that $K$ is tame in $L$ if for any $x \in L$ so that $v(x) \in \Gamma(K)$ there is a unique $y \in K$ so that $v(x-y)>\gamma$ for all $\gamma \in \Gamma(K)$.

With this definition we obtain the desired result for the p-adics. In the following proof we write $\mathbb{Q}_{p}^{a l g}$ for the prime model of $T h\left(\mathbb{Q}_{p}\right)$.

Proposition 4.2. $\mathbb{Q}_{p}$ is the unique p-adically closed field $K$ so that any elementary submodel is dense in $K$ and $K$ is tame in any elementary extension.

Proof. We first verify that $\mathbb{Q}_{p}$ satisfies the conclusions of the proposition. The fact that any elementary subfield of $\mathbb{Q}_{p}$ is dense is immediate since $\mathbb{Q}$ is dense in $\mathbb{Q}_{p}$. Next suppose that $\mathbb{Q}_{p} \preceq K$ and $x \in K$ with $v(x)=n \in \mathbb{Z}$. Note that we can find $a_{n} \in \mathbb{Q}_{p}$ so that $v\left(x-a_{n} p^{n}\right) \geq n+1$. Continuing we can find $a_{n+1}$ so that $v\left(x-a_{n} p^{n}-a_{n+1} p^{n+1}\right) \geq n+2$. Continuing in this manner we obtain a sequence $a_{i}$ with $i \geq n$. Let $a=\Sigma_{i \geq n} a_{i} p^{i} \in \mathbb{Q}_{p}$. Then $v(x-a)>n$ for all $n \in \mathbb{Z}$. For uniqueness suppose that there are distinct $b_{1}, b_{2} \in \mathbb{Q}_{p}$ such that $v\left(x-b_{1}\right)>n$ for all $n$ and $v\left(x-b_{2}\right)>n$ for all $n$. But then $v\left(x-b_{2}-\left(x-b_{1}\right)\right)=v\left(b_{1}-b_{2}\right)>n$ for all $n$ a contradiction.

Now suppose that $K$ is a $p$-adically closed field meeting our two criteria. We first claim that $\Gamma(K)=\mathbb{Z}$. Notice that by assumption $\mathbb{Q}_{p}^{\text {alg }} \preceq K$. Suppose that 
$\Gamma(K) \neq \mathbb{Z}$. Pick $\gamma \in \Gamma(K)$ so that $\gamma>n$ for all $n \in \mathbb{Z}$ and consider the open set $U=\{x \in K: v(x)>\gamma\} \backslash\{0\}$. Then $U \cap \mathbb{Q}_{p}^{a l g}=\emptyset$ a contradiction. So $\Gamma(K)=\mathbb{Z}$. Now fix $K^{*}$ a very saturated elementary extension of $K$ so that $\mathbb{Q}_{p} \preceq K^{*}$. By assumption for every $x \in \mathbb{Q}_{p}$ there is $y \in K$ so that $v(x-y)>n$ for all $n \in \mathbb{Z}$ and similarly for any $y \in K$ there is a unique $x \in \mathbb{Q}_{p}$ so that $v(x-y)>n$ for all $n \in \mathbb{Z}$. Hence we may define an isomorphism of $K$ to $\mathbb{Q}_{p}$ by simply sending $x \in K$ to the unique $y \in \mathbb{Q}_{p}$ so that $v(x-y)>n$ for all $n \in \mathbb{Z}$.

Given the previous result one is led, as in the case for real closed fields (or more generally o-minimal structures), to consider two situations for pairs of p-adically closed fields $\left(K_{1}, K_{2}\right)$ with $K_{2} \preceq K_{1}$, the first being where $K_{2}$ is dense in $K_{1}$ the second where $K_{2}$ is tame in $K_{1}$. In this note we will consider the first of these two cases.

Our goal is to generalize many of the result of [10] from the o-minimal context to the context of dense pairs of p-adically closed fields. But in fact we can work at a much greater level of generality and thus encompass a larger class of examples. Thus we now proceed to develop the machinery of dense pairs of o-minimal structures in a context of fields with a definable topology. Ultimately we will be able to show that much of the machinery from [10] carries over into this new context. This will allow us to use theorem 2.7 to show that if our initial theory is dependent the theory of the dense pairs we obtain is also dependent.

We begin by describing the context in which we will work. Our exposition relies heavily on that of Matthews in [18].

Definition 4.3. Let $\mathfrak{M}$ be an $\mathcal{L}$-structure and $\phi(x, \vec{y})$ be an $\mathcal{L}$ formula. The pair $(\mathfrak{M}, \phi)$ is called a first order topological structure if the set $\{\phi(M, \vec{a}): \vec{a} \in M\}$ forms the basis for a topology on $M$.

We will from now on assume that $\mathcal{L}$ contains symbols $\{+, \cdot, 0,1\}$ and any $\mathcal{L}$ structures considered are integral domains.

Definition 4.4. For $(\mathfrak{M}, \phi)$ a topological structure a definable set $X \subset M^{n}$ is a cell if for some coordinate projection $\pi: M^{n} \rightarrow M^{m}, \pi$ is a homeomorphism of $X$ onto its image and $\pi(X)$ is open.

Definition 4.5. The topological structure $(\mathfrak{M}, \phi)$ is said to have the cell decomposition property if for any $A \subset M$ and any A-definable $X \subset M^{n}$ there is a partition of $X$ into A-definable cells $X_{1} \ldots X_{n}$.

Notice that this is weaker than the cell decomposition property as defined in [18] in that we do not insist that a fixed function can be assumed to be continuous when restricted to a cell. The following definitions provides us with the class of first-order topological theories with which we will work.

Definition 4.6. Let $(\mathfrak{M}, \phi)$ be a topological structure the $T=T h(\mathfrak{M})$. We say that $(\mathfrak{M}, \phi(x, \vec{y}))$ is geometric if:

(1) T has definable Skolem functions.

(2) If $\mathfrak{N}=T$ then $(\mathfrak{N}, \phi)$ has the cell decomposition property.

(3) If $\mathfrak{N}=T$ then acl on $\mathfrak{N}$ satisfies the exchange property.

(4) If $\mathfrak{N}=T$ and $f: N \rightarrow N$ is definable then $f$ is continuous off of a finite subset of $N$.

(5) If $\mathfrak{N}=T$ and $X \subseteq N$ is definable and infinite then $X$ has interior. 
(6) If $p(x) \in M[x]$ is a polynomial with coefficients from $M$ then $p(x)$ is continuous.

(7) If $\vec{a} \in M^{n}$ (here $\left.n=|\vec{y}|\right)$ and $\mathfrak{M}=\phi(b, \vec{a})$ then there is $U$ a neighborhood of $\vec{a}$ so that if $\vec{c} \in U$ then $\mathfrak{M}=\phi(b, \vec{c})$.

Properties (5)-(7) above ensure that the definable topology and general definable sets interact in a reasonable way (although (5) is rather stringent) while properties (1)-(4) carry the actual weight that allow us to think of these theories as in some sense "geometric".

Recall that for any structure $\mathfrak{M}$ in which acl satisfies the exchange property we may define the $\operatorname{rank} r k(\vec{a} / A)$ of a tuple $\vec{a}$ over a set $A$. Thus we may define the dimension of an $A$-definable set $X \subseteq M^{n}$ as the maximal $m \in \mathbb{N}$ so that for some $\vec{a} \in X$ (after potentially passing to a sufficiently saturated elementary extension), $r k(\vec{a} / A)=m$. Also if $(\mathfrak{M}, \phi)$ is topological we may define, for $X \subseteq M^{n}$ definable, the dimension of $X$ to be the largest $l \in \mathbb{N}$ so that for some coordinate projection $\phi: M^{n} \rightarrow M^{l}, \pi(X)$ has interior. Under the assumptions that $(\mathfrak{M}, \phi)$ is a geometric topological structure it follows by Lemmas 8.11 and 8.12 in [18] that these two notions of dimension are the same and hence we are justified in simply speaking of dimension (we note that in [18] Lemmas 8.11 and 8.12 are proved under the stronger assumption for cell decomposition used in that paper, but for these two results our weaker definition suffices). Also notice that if $(\mathfrak{M}, \phi)$ is geometric then any locally constant definable $f: M \rightarrow M$ has finite image. Furthermore it follows from 1.18 and the ensuing remarks in [9] that if $T$ is geometric then it eliminates $\exists^{\infty}$. Finally the assumption that $T$ has Skolem functions yields that for any $\mathfrak{M} \models T$ if $A \subset M$ then $\operatorname{dcl}(A)=\operatorname{acl}(A)$ hence these two notions can be used interchangeably.

We now develop the dense pairs machinery of [10] in the context of geometric topological theories. Our exposition is almost identical to that of [10]. We recall some definitions.

Definition 4.7. Given a first order topological theory $T$ (recall our conventions on $T$ ) the theory of dense pairs of models of $T, T^{d}$ is the theory of pairs of model of $T,(\mathfrak{N}, \mathfrak{M})$ where $\mathfrak{M} \prec \mathfrak{N}$ and $M$ is dense in $N$.

Definition 4.8. If $(\mathfrak{N}, \mathfrak{M}) \models T^{d}$, a definable set $X \subseteq N$ is called small if there is an $\mathfrak{N}$-definable function $f: N^{m} \rightarrow N$ so that $X \subseteq f\left(M^{m}\right)$.

We now summarize the results we intend to develop for dense pairs of geometric topological theories. Essentially this theorem summarizes theorems (1)-(4) in [10] with very modest alterations for the broader context. In the ensuing Theorem, we write $(\mathcal{B}, \mathcal{A})$ instead of $(\mathfrak{N}, \mathfrak{M})$ in order for our notation to coincide with that of [10].

Theorem 4.9. It $T$ is a geometric topological theory and $T^{d}$ is the theory of dense pairs of models of $T$ then:

(1) $T^{d}$ is complete and any formula is $T^{d}$ equivalent to a Boolean combination of formulas of the form:

$$
\exists \vec{x}(P(\vec{x}) \wedge \phi(\vec{x}, \vec{y}))
$$

where $\phi$ is an $\mathcal{L}$ formula.

(2) If $(\mathcal{B}, \mathcal{A}) \models T^{d}$ then $Y \subseteq A^{m}$ is definable in $(\mathcal{B}, \mathcal{A})$ if and only if $Y=Z \cap A^{m}$ for some $Z \subseteq B^{m}$ definable in $\mathcal{B}$. 
(3) If $F: B \rightarrow B$ is definable in $(\mathcal{B}, \mathcal{A})$ then $F$ agrees off of some $A$-small subset of $\mathcal{B}$ with a function definable in $\mathcal{B}$.

(4) If $S \subseteq B$ is definable in $(\mathcal{B}, \mathcal{A})$ then there exists a small subset $X \subseteq B$ so that $S \backslash X=S^{\prime} \backslash X$ for some $\mathcal{B}$-definable $S^{\prime} \subseteq B$.

(5) If $f: A^{n} \rightarrow A$ is definable in $(\mathcal{B}, \mathcal{A})$ then there are $f_{1} \ldots f_{l}: A^{n} \rightarrow A$ definable in $\mathcal{A}$ so that for each $x \in M^{n}$ there is $1 \leq i \leq l$ so that $f(x)=$ $f_{i}(x)$.

(6) If $X \subseteq B$ is $A$-small and definable then there are definable open sets $U, V$ so that $B \backslash(U \cup V)$ is finite, $U \cap X=\emptyset$ and $X$ is dense and codense in $V$. If $S \subseteq B$ is definable in $(\mathcal{B}, \mathcal{A})$ then there are definable open sets $U, V, W$ so that $N \backslash(U \cup V \cup W)$ is finite, $U, V, W$ are pairwise disjoint, $U \subseteq S$, $S \cap V=\emptyset$, and $S \cap W$ is dense and co-dense in $W$.

Proof. The proof of this theorem is almost identical to those found in [10]. We do not intend to rewrite all of van den Dries's arguments, rather we point out any places in the exposition of [10] where significant alterations are needed for our context. Throughout we refer to the numbering found in [10]. Our goal is to verify results 1.1-4.6 in [10] in our context. We provide a guide to the alterations necessary to generalize van den Dries's proof. We assume $T$ is a first order topological theory witnessed by a formula $\phi(x, \vec{y})$. Notice that throughout where [10] has "cut of $a$ over $B$ " we must rephrase this as " $t p_{\mathcal{L}}(a / B)$ ". Similarly throughout we must replace "interval" with "basic open set".

Results 1.1,1.3, and 1.4 follow immediately, notice we do not consider 1.2 because we assume that we are working in structures which are at least integral domains. Once again 2.1 and 2.3 follow immediately and we omit 2.2 (which is not necessary for the rest of the results). We need a new proof for 2.4:

Claim. $B \backslash A$ is dense in $B$.

Suppose that $\mathcal{A} \prec \mathcal{B}$. For contradiction suppose that for some $\vec{b} \in B^{m}$ a basic open set $U=\phi(B, \vec{b})$ is a subset of $A$. Since acl is a pregeometry it follows from 1.18 and the ensuing remarks in [9] that there is a $\emptyset$-definable function $F: B^{4} \rightarrow B$ in the language of rings so that $F(U)=B$, but since $\mathcal{A} \models T, U \subseteq A$, and $f$ is $\mathcal{L}$-definable over $\emptyset$ it follows that $F(U) \subseteq A$. Thus $B=A$ a contradiction.

Given this 2.5-2.10 follow mutatis mutandis except that for 2.11 we only consider the equivalence of statements (1) and (2), (3) of course being only relevant in the ominimal case. Similarly 3.1 and 3.2 also follow. We must make a slight adjustment to verify 3.3 :

Claim. Let $(\mathcal{B}, \mathcal{A}) \subset\left(\mathcal{B}^{*}, \mathcal{A}^{*}\right) \models T^{p}$ and suppose that $\mathcal{B}$ and $\mathcal{A}^{*}$ are algebraically independent over $\mathcal{A}$. Then $\mathcal{B}$ is definably closed in $\left(\mathcal{B}^{*}, \mathfrak{A}^{*}\right)$.

The proof of 3.3 remains the same except the we may not assume upon cell decomposing and choosing an open cell $E$ that $f \uparrow E$ is monotone or constant. Notice that Case 1 of the proof of 3.3 from [10] holds hence we may assume that $b^{*}$ depends on all of $a_{1}^{*} \ldots a_{k}^{*}$. As in the original proof we choose by saturation $a_{k}$ so that $\operatorname{tp}_{\mathcal{L}}\left(a_{k} / B^{\prime}\right)=t p_{\mathcal{L}}\left(a_{k}^{*} / B^{\prime}\right)$ and so that $a_{k} \notin \operatorname{acl}_{\mathcal{L}}\left(B a_{1}^{*} \ldots a_{k}^{*} b^{*}\right)$. But we have that $b^{*} \in \operatorname{acl}\left(B a_{1}^{*} \ldots a_{k-1}^{*} a_{k}\right)$ and thus by the exchange property we have that $a_{k} \in \operatorname{acl}_{\mathcal{L}}\left(B a_{1}^{*} \ldots a_{k-1}^{*} b^{*}\right)$ a contradiction.

With this 3.4-3.6 follow as does 4.1. 4.2 is the last result in which we need to make minor modifications. 
Claim. Let $S \subset B^{m}$ be definable in $\mathcal{B}$, and let the map $g=\left(g_{1}, \ldots, g_{k}\right): B^{m} \rightarrow$ $B^{k}$ be definable in $\mathcal{B}$. Then there is a set $S^{\prime} \subset S$ definable in $\mathcal{B}$ such that

$$
A^{m} \cap S \cap g^{-1}\left(A^{k}\right)=A^{m} \cap S^{\prime}
$$

Our proof parallels that of [10] to the point where we take a function $G: B^{m+n} \rightarrow$ $B$ which is $A$-definable in $B$ and a point $b=\left(b_{1} \ldots b_{n}\right) \in B^{n}$ so that $g(x)=G(x, b)$ for all $x \in B^{m}$. We also assume that $b_{1} \ldots b_{n}$ are independent over $A$ and following [10] proceed by induction on $n$. We may also assume that $n>0$. We now take a cell decomposition of $B^{m+n}$. By induction we may without loss of generality restrict our attention to open cells $E$. After further cell decomposition we may also assume that either for any $a_{1} \ldots a_{n+m} \in E, G\left(a_{1} \ldots a_{n+m-1}, x\right)$ is locally constant in a neighborhood of $a_{n+m}$ or for no $a_{1} \ldots a_{n_{m}} \in E$ is $G\left(a_{1} \ldots a_{n+m-1}, x\right)$ locally constant in a neighborhood of $a_{n+m}$. Thus we divide into two cases paralleling cases (1) and (2) of the proof of 4.3 in [10]

Suppose we are in the first case. Let $E^{*}$ be the projection of $E$ onto the first $n+m-1$ coordinates. Notice that for any $a_{1} \ldots a_{n+m-1} \in E^{*}$ the function $G\left(a_{1} \ldots a_{n+m-1}, x\right)$ obtains only finitely many values on the fiber of $E$ above $a_{1} \ldots a_{n+m-1}$ and hence there is uniform bound on the number of values, $N$. Since we have Skolem functions we may find functions $G_{1} \ldots G_{N}: E^{*} \rightarrow B$ which pick out these values. Now let $E_{i}$ be the set of all $a_{1} \ldots a_{n+m} \in E$ so that $G\left(a_{1} \ldots a_{n+m-1}, x\right)=G_{i}\left(a_{1} \ldots a_{n+m-1}\right)$ in some neighborhood of $a_{m+m}$. Thus $E=\cup E_{i}$ and $G$ is independent of the last coordinate on each $E_{i}$. Now we may apply cell decomposition to each of the $E_{i}$ and proceed as in case 1 of the proof of 4.2 in [10].

Now suppose we are in the second case. Let $a_{1} \ldots a_{n+m} \in E$. Notice that the set $E\left(a_{1} \ldots a_{n+m}\right)$ of all $z \in B$ so that $a_{1} \ldots a_{n+m-1}, z \in E$ and $G\left(a_{1} \ldots a_{n+m}\right)=$ $G\left(a_{1} \ldots a_{n+m-1}, z\right)$ must be finite since otherwise $E\left(a_{1}, \ldots a_{n+m}\right)$ would have interior, contradicting the assumptions on $E$. By elimination of $\exists^{\infty}$ for $T$ we may find a uniform bound, $N$, on the cardinality of $E\left(a_{1}, \ldots a_{n+m}\right)$ as we vary $a_{1} \ldots a_{n+m}$. We may find functions $G_{1}, \ldots, G_{N}$ so that if $a_{1} \ldots a_{n+m} \in E$ and $G\left(a_{1} \ldots a_{n+m}\right)=c$ then

$$
G_{1}\left(a_{1} \ldots a_{n+m-1}, c\right) \ldots G_{N}\left(a_{1} \ldots a_{n+m-1}, c\right)
$$

lists all elements $z$ so that $a_{1} \ldots a_{n+m-1}, z \in E$ and $G\left(a_{1}, \ldots a_{n+m-1}, z\right)=c$. Thus if $a_{1} \ldots a_{m} \in M^{m} \cap S_{E} \cap g^{-1}(A)$ then $G\left(a_{1} \ldots a_{m}, b\right)=a^{\prime} \in A$ so that $b_{n}=$ $G_{i}\left(a_{1}, \ldots a_{m} b_{1}, \ldots b_{n-1} a^{\prime}\right)$ for some $i$ contradicting that $b_{n} \notin \operatorname{acl}\left(M b_{1} \ldots b_{n-1}\right)$. Hence $A^{m} \cap S_{E} \cap g^{-1}(A)$ is empty.

Now 4.3-4.6 go through mutatis mutandis.

Corollary 4.10. If $T$ is a geometric first-order topological theory without the independence property then the theory of dense pairs of models of $T$ also does not have the independence property. Moreover if $T$ is strongly dependent so is the theory of dense pairs of models of $T$.

Proof. The previous theorem establishes that if $\mathfrak{N} \models T$ and $\mathfrak{M}$ is a dense elementary submodel then $M$ is an innocuous subset of $N$ so the result is immediate from Theorem 2.7. For the "moreover" we apply Theorem 2.10 instead.

Corollary 4.11. For any of the following theories, the resulting theory of dense pairs of models of $T$ is strongly dependent: 
(1) O-minimal theories.

(2) P-minimal theories with Skolem functions. Examples include: $T h\left(\mathbb{Q}_{p}\right)$ and $T h\left(\mathbb{Q}_{p}^{a n}\right)$ the theory of the p-adics with restricted analytic functions (for which see [13]).

(3) Weakly o-minimal theories with Skolem functions for which acl-satisfies exchange. In particular the theory of real closed rings (see [18] and [17]).

Proof. The result follows in that each of the above theories is a geometric first order dependent topological theory. For the fact that each of these is geometric: see [18] for the o-minimal case as well as for the p-adics, for the weakly o-minimal case see [17] and [18] for specifics on real closed rings, see [16] for generalities on p-minimal theories, and [13] for specific facts on $\mathbb{Q}_{p}^{a n}$. For the strong dependence in the weakly o-minimal (and hence o-minimal case) see [14] where even stronger results than strong dependence are proved. For the p-minimal case see [22] for the strong dependence of the p-adics and then argue as in [16] for the general p-minimal case.

\section{REFERENCES}

[1] Hans Adler, A geometric introduction to forking and thorn-forking, preprint. available at www.amsta.leeds.ac.uk/ adler.

[2] B. Baizhanov and J. Baldwin, Local Homogeneity, Journal of Symbolic Logic, 69, (2004), pp. 1243-1260.

[3] I. Ben Yaacov, A. Pillay, E. Vassiliev, Lovely pairs of models, Annals of pure and applied logic 122 (2003), pp. 235-261.

[4] A. Berenstein, Lovely pairs and dense pairs of O-minimal structures, submitted, 2007. available at www.logique.jussieu.fr/modnet/Publications/Preprint $\% 20$ server/

[5] G. Boxall, Lovely pairs and dense pairs of real closed fields, Ph.D. Thesis, University of Leeds.

[6] S. Buechler, Pseudoprojective Strongly Minimal Sets are Locally Projective, Journal of Symbolic Logic, 56, (1991), pp. 1184-1194

[7] E. Casanovas and M. Ziegler, Stable theories with a new predicate, Journal of Symbolic Logic, 66, (2001), pp 1127-1140.

[8] Z. Chatzidakis and A. Pillay, Generic structures and simple theories, Annals of Pure and Applied Logic, (1998), pp. 71-92.

[9] A. Dolich, C. Miller, C. Steinhorn, Structures having o-minimal open core, to be published in Transactions of the American Mathematical Society.

[10] L. van den Dries, Dense pairs of o-minimal structures, Fund. Math. 157 (1998), pp. 61-78.

[11] L. VAn Den Dries, Tame topology and o-minimal structures, London Mathematical Society Lecture Notes Series, 248, Cambridge University Press, Cambridge, 1998.

[12] L. van den Dries and A. Lewenberg, T-convexity and tame extensions, The Journal of Symbolic Logic, 60, (1995), pp. 74-102.

[13] J. Denef and L. van den Dries, P-adic and real subanalytic sets, The Annals of Mathematics, 128, (1988), pp. 79-138.

[14] J. Goodrick,A Monotonicity Theorem for Dp-minimal Densely Ordered Groups, preprint. available at www.math. umd.edu/ goodrick

[15] A. Günaydin and P. Hieronymi, Dependent pairs, preprint.

[16] D. Haskell and D. Macpherson, A version of o-minimality for the p-adics, The Journal of Symbolic Logic, 62, (1997), pp. 1075-1092.

[17] D. Macpherson, D. Marker, C. Steinhorn, Weakly o-minimal structures and real closed fields, Transactions of the American Mathematical Society, 352, (2000), pp. 5435-5483.

[18] L. Matthews, Cell decomposition and dimension functions in first-order topological structures, Proceedings of the London Mathematical Society. s3-70 (1995), pp 1-32.

[19] A. Onshuus, Properties and consequences of thorn-independence, Journal of Symbolic Logic, 71 (2006), pp 1-21.

[20] B. Poizat Paires de Structures Stables, Journal of Symbolic Logic, 48 (1983), pp 239-249. 
[21] S. Shelah, Dependent first order theories, continued, SH783. available at shelah.logic.at/

[22] S. Shelah, Strongly Dependent Theories, SH863. available at shelah.logic.at/

[23] A. Pillay On externally definable sets and a theorem of Shelah, preprint. available at www.amsta.leeds.ac.uk/ ${ }^{\sim}$ pillay/

[24] E. VAssiliev, Generic pairs of SU-rank 1 structures, Annals of Pure and Applied Logic, 120, (2003), pp. 103-149.

Universidad de los Andes, Cra 1 No 18A-10, Bogotá, Colombia

E-mail address: aberenst@uniandes.edu.co

$U R L$ : matematicas .uniandes.edu.co/ aberenst

Chicago State University, 9501 South King Drive, Chicago, Illinois 60628-1598, USA

E-mail address: adolich@csu.edu

$U R L$ : www. dolich.com

Universidad de los Andes, Cra 1 No 18A-10, Bogotá, Colombia

E-mail address: aonshuus@uniandes.edu.co 